INRA Prod. Anim., 2008, 21 (1), 59-70

\title{
Les conduites de traite simplifiées en élevage laitier : vers la levée de l'astreinte biquotidienne
}

\author{
D. POMIĖS', P.-G. MARNET'2, S. COURNUT'3 , F. BARILLET4, \\ J. GUINARD-FLAMENT' ${ }^{2}$, B. REMOND ${ }^{5}$ \\ ${ }^{1}$ INRA, UR1213 Herbivores, F-63122 Saint-Genès-Champanelle, France \\ 2 INRA, Agrocampus, UMR1080 Production de lait, F-35590 Saint-Gilles, France \\ ${ }^{3}$ INRA, AgroParisTech, CEMAGREF, ENITAC, UMR1273 Metafort, F-63370 Lempdes, France \\ ${ }^{4}$ INRA, UR631 Amélioration Génétique des Animaux, F-31326 Castanet-Tolosan, France \\ 5 INRA, ENITAC, USC2005, Élevage et Production des Ruminants, F-63370 Lempdes, France \\ Courriel :dominique.pomies@clermont.inra.fr
}

Face aux mutations récentes de la société française, la réduction du travail est devenue la principale aspiration des éleveurs laitiers. Depuis plus de dix ans l'INRA met au point et/ou évalue des pratiques de traite et des outils (robot de traite) innovants, qui permettent de réduire de façon importante l'astreinte de la traite biquotidienne.

Le secteur de l'élevage laitier n'a pas échappé aux tendances d'agrandissement des structures et de diminution de la main-d'œuvre familiale qui caractérisent l'évolution de l'agriculture depuis plus de trente ans. Selon le RICA (Réseau d'Information Comptable Agricole), le nombre de vaches laitières par unité de travail agricole a augmenté de $30 \%$ entre 1988 et 2000. Dans les élevages caprins, sur la même période, la tendance est encore plus marquée : l'effectif par troupeau a progressé de $114 \%$ à l'échelle française, et même de $200 \%$ dans des départements à dominante d'éleveurs-livreurs comme les Deux-Sèvres. En 2005, on comptait $48 \%$ des animaux dans des structures d'environ 300 chèvres (Agreste, Ministère de l'agriculture et de la pêche). Dans cette filière, dont près de la moitié des élevages sont conduits par des éleveurs-fromagers, le temps de travail est un vrai frein à l'accroissement des structures qui, de fait, ne dépassent que rarement les 70 chèvres. Chez les éleveurs ovins laitiers l'évolution a même été plus rapide, avec une spécialisation par grands bassins de production qui a rendu la population d'élevages plus homogène, avec environ 250 brebis laitières par exploitation en 2005, dont $27 \%$ dans des élevages de plus de 500 animaux.
L'élevage laitier est également touché de plein fouet par les mutations sociologiques qui affectent le rapport des agriculteurs au travail : les attentes d'un travail maîtrisé, distinct de la vie familiale, et la volonté de se préserver du temps libre prennent le pas sur le «labeur paysan» (Barthez 1986), où vie privée et travail sont confondus. La problématique du travail en élevage laitier est exacerbée par la traite biquotidienne, qui représente toujours près de $50 \%$ de l'astreinte quotidienne des éleveurs contre $33 \%$ pour l'alimentation (Chauvat et al 2003). Le travail n'est pas qu'un simple facteur de production, il participe aussi à la construction d'un projet de vie associé à l'exercice du métier d'éleveur (Dedieu et al 2006). D'une façon générale, les solutions explorées par les éleveurs pour résoudre leurs problèmes de travail relèvent de trois ensembles (Dedieu et Servière 2004) : la recomposition $\mathrm{du}$ collectif de travail (association d'exploitations, groupements d'employeurs...), l'amélioration des bâtiments et des équipements (robot de traite, par exemple) et la conduite de l'élevage (dont la traite). La conduite de la traite est donc apparue de longue date comme un levier essentiel, d'autant que des systèmes simplifiés, largement utilisables, peuvent autant servir à réduire la charge de travail et améliorer les conditions de vie sociale (demande croissante des pays à coût de maind'œuvre élevé) qu'à accroître, dans certains cas, la productivité du travail.

Cette revue présente les récents travaux de l'INRA sur les conduites de traite simplifiées, en termes de choix, de réponse aux attentes des éleveurs, d'évaluation de leur impact (zootechnique, technologique, économique...) sur les systèmes d'élevage et d'étude des mécanismes. Parmi ces techniques, nous aborderons la traite robotisée, initialement conçue dans une logique d'intensification de la production avec une multiplication des traites quotidiennes, mais qui permet surtout un allègement de la charge de travail et dispense théoriquement l'éleveur d'être présent à la traite. A l'opposé, nous étudierons des techniques qui reposent sur une baisse de la fréquence de traite, telles que la monotraite (une seule traite par jour à intervalle de $24 \mathrm{~h}$ ), la suppression de la traite du dimanche soir, les traites à rythmes irréguliers (3 traites en 2 jours et traites à intervalles très inégaux) et la conduite mixte «monotraite + tétée», où la traite n'est réalisée qu'une fois par jour mais compensée par une période de tétée qui limite les contraintes d'alimentation des jeunes. 


\section{1 / Pourquoi adopter des conduites de traite simpli- fiées ? Qui est concerné ? Quelles sont les implica- tions sur les systèmes tech- niques?}

Comme le souligne l'enquête de Guillaumin et al (2005), les éleveurs ont une perception du travail qui ne met pas en avant que la durée du travail ou la pénibilité de certaines tâches, mais exprime aussi des attentes portant sur les rythmes de travail : week-end libérés $(53 \%)$, vacances $(41 \%)$ et disponibilité accrue au quotidien (29\%). De récentes études (Cournut et Dedieu 2005, Rubin et al 2006) confirment ces observations et montrent que les adaptations mises en place par les éleveurs ne répondent pas aux mêmes attentes. Elles peuvent ainsi renvoyer à la volonté de réduire la durée du travail d'astreinte quotidien ou donner de la souplesse à ce travail (monotraite, robot, conduite mixte «monotraite + tétée»), de dégager du temps lors d'une période de l'année où l'interaction avec les loisirs ou d'autres tâches agricoles pose des problèmes (monotraite périodique), ou d'introduire une arythmie hebdomadaire comme dans la plupart des professions, où le dimanche est un jour à part (suppression de la traite du dimanche soir).

Ces adaptations ne s'insèrent pas de la même façon dans les systèmes techniques. Nous avons ainsi montré (Cournut et Dedieu 2005) que la suppression de la traite du dimanche soir n'a pas remis en cause les logiques de conduite, même si les éleveurs concernés sont plutôt des éleveurs à bon niveau de productivité. La monotraite pratiquée de façon saisonnière apparaît comme une solution souple qui peut avoir des répercussions plus ou moins grandes sur l'organisation du système : elle permet la diminution des apports d'aliments concentrés et l'augmentation de la part de pâturage à certaines périodes, la valorisation de parcelles éloignées, et peut conduire au regroupement des vêlages. Dans ce cas, la modification des pratiques de reproduction peut fortement influer sur la logique de conduite (réformes plus sévères sur des critères d'infertilité par exemple). Rubin et al (2006) montrent aussi que la mise en œuvre de la monotraite ou l'adoption du robot de traite n'ont pas les mêmes conséquences sur le système fourrager et le mode d'utilisation de la prairie. Il y a bien, tant sur le plan des conséquences au niveau du travail que sur celui des implications techniques, une grande variété de contenus regroupés sous le terme plutôt vague de «simplification des techniques de traite».

Enfin les choix de simplification ne peuvent être réfléchis sans prendre en compte la nature du collectif de personnes qui organisent et assurent le travail de l'exploitation, et de leur conception du travail (Dufour et al 2007). Ainsi la traite reste pour beaucoup d'éleveurs un poste clé qu'il est difficile de déléguer à un «étranger», ou qui met en jeu les relations qu'ils entretiennent avec leurs animaux. Toute simplification n'est pas possible ou envisageable pour tous.

\section{2 / Le robot de traite}

Le premier prototype opérationnel de robot de traite a été construit aux Pays-Bas en 1988. Il a été suivi dans la plupart des pays de l'Union Européenne de la commercialisation par Prolion (1992) et Lely (1994) de modèles destinés aux éleveurs. L'INRA s'est rapidement intéressé à cette machine, et un robot de traite a été installé dès octobre 1997 à l'Unité Expérimentale des Monts Dore, avec 2 objectifs : 1/ pouvoir modifier à souhait le rythme et les horaires de traite dans les études en cours sur la modulation de la courbe de lactation (Rémond et Boit 1997) ; 2/ préparer les réponses aux questions que ne

Photo 1. Le robot de traite de I'Unité Expérimentale des Monts Dore.

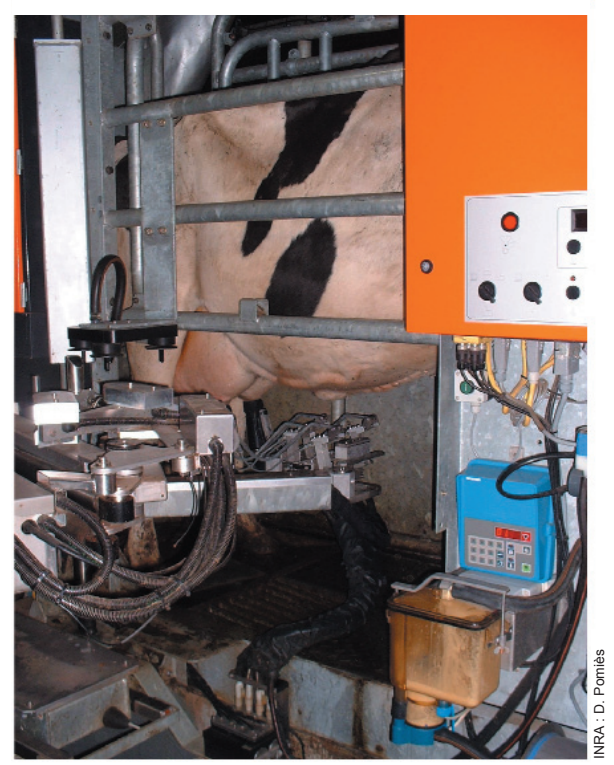

manqueraient pas de se poser les éleveurs et les acteurs de la filière sur cette technologie, et étudier les interactions vache-robot. A cette fin, un groupe de travail pluridisciplinaire composé de zootechniciens, physiologistes, économistes et spécialistes de la reproduction ou du comportement de l'INRA, a été mis en place pour travailler sur cette machine.

\section{1 / Limites d'utilisation du robot de traite et adaptation des animaux}

Après une première année d'utilisation, il s'est avéré que le robot de traite ne pouvait servir d'outil expérimental pour le programme de recherches sur les rythmes de traite. En effet, le taux d'échec de pose des gobelets trayeurs était élevé pour des fréquences de traite demandées inférieures à 2 fois par jour chez des vaches multipares en début de lactation (mamelle très distendue) ou supérieures à 2 fois par jour chez des vaches primipares en fin de lactation (trayons arrières croisés par insuffisance de lait dans la mamelle). Les tout premiers résultats (Pomiès et al 1998) ont mis en évidence la nécessité de réformer environ $15 \%$ des primipares pour des problèmes de conformation des mamelles (trayons trop courts, notamment), mais aussi une rapide adaptation des vaches à la machine et une variabilité de la production laitière journalière deux fois plus élevée qu'en salle de traite (en relation avec des intervalles de temps irréguliers entre traites). Des observations ont montré qu'une circulation «dirigée» des animaux (couchage $\rightarrow$ robot $\rightarrow$ alimentation $\rightarrow$ couchage) permettait une fréquentation continue (jour et nuit) du robot, avec des vaches passant $50 \%$ de leur temps couchées dans les logettes, $30 \%$ dans l'aire d'alimentation (dont la moitié à manger) et $2 \%$ dans le box de traite (Bony 2002), données équivalentes à celles relevées en stabulation libre et salle de traite classique.

\section{2 / Physiologie de la traite}

Comparativement à la traite classique, la traite robotisée présente des durées de traite et des débits de lait (moyen et maximum) identiques (Marnet 2002). Cependant, au robot, un pourcentage supérieur de lait résiduel a été mesuré $(8,7 \%$ vs $6,5 \%$; $\mathrm{p}<0,05)$ ainsi qu'un nombre de courbes d'émission du lait bimodales plus élevé $(>25 \%)$, suggérant un réflexe d'éjection du lait plus souvent perturbé. Ces données ont été confirmées par des 
pics de décharge d'ocytocine significativement inférieurs ( 73 vs 108 pg/mL) et des quantités totales d'ocytocine plasmatique déchargées inférieures. Ces résultats, relatifs à un seul type de robot et de salle de traite (avec leurs réglages propres), suggèrent donc une moindre capacité de stimulation physiologique du robot par rapport à une préparation manuelle de la mamelle en salle de traite, mais des données plus récentes obtenues sur d'autres modèles de robot semblent montrer une amélioration de ce point.

\section{3 / Production laitière et com- position du lait}

L'augmentation de la production laitière des vaches permise par la fréquence de traite plus élevée du robot a souvent constitué un argument de vente des fabricants. Nous l'avons étudiée lors d'un essai croisant le type de machine (robot $v s$ salle de traite) et le nombre de traite quotidien (2 vs 3) (Pomiès et Lefeuvre 2001). Si le passage de 2 à 3 traites par jour a effectivement permis une augmentation de la production laitière $(+1,8 \mathrm{~kg} / \mathrm{j}$; $\mathrm{p}<0,001)$, cet avantage a été fortement réduit par l'effet propre du robot $(-1,3 \mathrm{~kg} / \mathrm{j} ; \mathrm{p}<0,01)$, conséquence de l'irrégularité des intervalles de traite, des difficultés de pose du faisceau trayeur et du système de nettoyage des trayons (spécifique du modèle utilisé) qui élimine le lait de début de traite avec l'eau de lavage. La qualité du lait a principalement été étudiée par comparaison des analyses des prélèvements effectués toutes les semaines dans les deux tanks associés aux deux systèmes de traite (robot $v S$ salle de traite) de l'Unité Expérimentale des Monts Dore, durant 5 hivers consécutifs (tableau 1, Pomiès 2002). Si le point de congélation du lait et le nombre de cellules somatiques ne semblent pas affectés par le mode de traite, on observe avec le robot un doublement des germes totaux (vraisemblablement lié à une présence de lait permanente dans des circuits souvent plus complexes), des spores butyriques (lié à un lavage des trayons insuffisant sur les vaches sales) et des acides gras libres (lié à des traites plus fréquentes et à un «barattage» plus important). Néanmoins, seule la concentration en spores butyriques a parfois entraîné des pénalités dans le paiement du lait.

\section{4 / Reproduction}

Avec un robot, la moindre présence de l'éleveur auprès de ses vaches pouvait faire craindre une moins bonne efficacité de la détection des chaleurs et donc une incidence sur les paramètres de reproduction. Afin de rassembler des éléments de réponse, des mesures ont été réalisées lors de la mise en service du robot des Monts Dore (Saumande 2002). Il en est ressorti qu'en système traditionnel, 52\% des oestrus sont observés par l'éleveur en salle de traite, contre $11 \%$ quand l'éleveur s'occupe du robot, dans l'autre système. Dans l'étable équipée du robot, une grande partie des chaleurs (36\%) a été enregistrée lors de 2 périodes d'observation de $30 \mathrm{~min}$ spécialement dédiées. Malgré cela, la durée moyenne des cycles (estimée par l'observation des chaleurs) a été de 38 jours au robot contre 25 jours en salle de traite, ce qui traduit une diminution de l'efficacité de la détection des œstrus lors de la mise en place d'un robot, dont éleveurs et fabricants semblent peu se soucier.

\section{5 / Enquête auprès des pre- miers éleveurs équipés d'un robot de traite}

Parallèlement aux expérimentations menées sur la machine des Monts Dore, une enquête a été réalisée en 2000 par l'INRA auprès de 44 éleveurs, soit près de la moitié des exploitations françaises alors équipées d'un robot de traite

Tableau 1. Comparaison des analyses de lait des tanks associés aux deux systèmes de traite de l'Unité Expérimentale des Monts Dore, durant 5 hivers consécutifs (19972001).

\begin{tabular}{|l|c|c|c|c|}
\hline & $\begin{array}{c}\text { Nombre } \\
\text { d'analyses }\end{array}$ & $\begin{array}{c}\text { Tank } \\
\text { «salle de traite» }\end{array}$ & $\begin{array}{c}\text { Tank } \\
\text { «robot» }\end{array}$ & \\
\hline Germes totaux $(\times 1000 / \mathrm{mL})$ & $2 \times 200$ & 9 & 19 & $* * *$ \\
\hline Spores butyriques $(\mathrm{L})$ & $2 \times 150$ & 558 & 1122 & $* * *$ \\
\hline $\begin{array}{l}\text { Acides Gras Libres } \\
(\mathrm{meq} / 100 \mathrm{~g} \mathrm{MG})\end{array}$ & $2 \times 130$ & 0,32 & 0,61 & $* * *$ \\
\hline Cryoscopie $\left({ }^{\circ} \mathrm{C}\right)$ & $2 \times 180$ & $-0,525$ & $-0,526$ & $*$ \\
\hline $\begin{array}{l}\text { Nombre de cellules somatiques } \\
(\times 1000 / \mathrm{mL})\end{array}$ & $2 \times 200$ & 211 & 204 & $\mathrm{~ns}$ \\
\hline
\end{tabular}

${ }^{* * *}: p<0,001 ;{ }^{*}: p<0,05 ; n s: p \geq 0,05$.
(Veysset et al 2001). Il en est ressorti que les acheteurs de robots se situaient majoritairement dans des zones de polyculture-élevage (Pays de Loire, Bretagne, Lorraine...), dans des structures importantes (77 vaches en moyenne), aux surfaces et à la production de lait largement supérieures à la moyenne nationale, ayant recours à de la main-d'œuvre salariée $(16 \%$ des UTH). Pour ces éleveurs, les principaux critères avancés pour l'achat d'un robot sont la réponse à un problème de main-d'œuvre (91\%), la volonté de diminuer leur charge de travail $(80 \%)$ et de mieux organiser ce travail (souplesse horaire : $52 \%$; week-ends et loisirs : 41\%). Après plus de 6 mois d'utilisation, ces 44 éleveurs estiment avoir diminué la charge et la pénibilité de leur travail $(86 \%)$ et surtout avoir gagné en souplesse horaire $(86 \%)$. En contrepartie, le robot a souvent modifié la conduite estivale du troupeau (passage d'un pâturage «vrai» à un pâturage «limité» et progression de $63 \%$ du «zéro pâturage»). D'après les simulations réalisées sur 3 exploitations types de 60, 80 et 100 vaches laitières (calcul des budgets partiels de l'atelier lait avec détermination du surcoût par rapport à une salle de traite neuve toutes options), celles les plus aptes à rentabiliser économiquement un robot sont des structures familiales de moins de 65 animaux. Au-delà, la capacité limitée d'un robot monostalle nécessite l'installation d'une $2^{\text {ème }}$ machine dont le prix élevé (environ $200000 €$ au total) entre en concurrence avec la main-d'œuvre salariée.

Malgré des avancées technologiques permanentes, une croissance toujours soutenue des ventes (environ 8000 robots dans le monde fin 2007, dont 800 en France) et une restructuration des acteurs du marché, ces résultats restent d'actualité et ont été confirmés dans une récente synthèse (Billon et Pomiès 2006). Si le robot de traite représente l'avenir d'une partie de la production laitière, dans les pays où le coût de la main-d'œuvre est élevé, son prix devra nécessairement baisser pour qu'il puisse s'adresser aux éleveurs les plus concernés par les problèmes d'astreinte. De plus, des progrès techniques dans la détection des laits anormaux et des solutions pour mieux concilier robot de traite et pâturage sont impératifs, afin de préserver auprès des consommateurs-citoyens l'image de qualité du produit «lait» et celle de bien-être des vaches. 


\section{3 / La monotraite}

La traite des vaches laitières une seule fois par jour (ou monotraite) est une conduite qui a vu le jour dans les années 80 en Nouvelle-Zélande, dans un contexte très particulier. Avec des animaux produisant moins de $5000 \mathrm{~kg}$ de lait par an et entièrement conduits au pâturage, son utilisation durant les 2-3 derniers mois de lactation entraîne une baisse de production relativement faible, mais permet à des exploitations de taille importante de réduire de près de moitié le coût de la main-d'œuvre salariée (Davis et al 1999). En France, les premiers essais sur bovins ont eu lieu à l'INRA (UE des Monts Dore) dès 1996 (Rémond et al 1999), dans l'optique d'écrêter le pic de lactation et, ainsi, de réduire la quantité d'aliments concentrés distribuée aux animaux durant cette période sans risque de déséquilibre nutritionnel. Mais son utilisation pour diminuer l'astreinte des éleveurs s'est rapidement imposée, et les travaux se sont orientés sur les conséquences de cette pratique (bien-être des animaux, production laitière, qualité du lait et des fromages, reproduction, pathologie, économie de l'élevage...), chez les bovins comme chez les petits ruminants. Les études ont également porté sur les facteurs de variation de la réponse à la monotraite et sur l'analyse des mécanismes mis en jeu.

\section{1 / Comportement et bien-être}

De l'avis même des animaliers de l'INRA, la monotraite ne semble pas poser de problème aux vaches. Les différents essais réalisés dès le vêlage montrent que les animaux manifestent peu de signes d'inconfort ou de comportements particuliers (Rémond et al 1999, 2002, 2004). Tout au plus notet-on durant les 3 premières semaines quelques écoulements de lait des

Photo 2. Vaches en monotraite à l'Unité Expérimentale des Monts Dore.

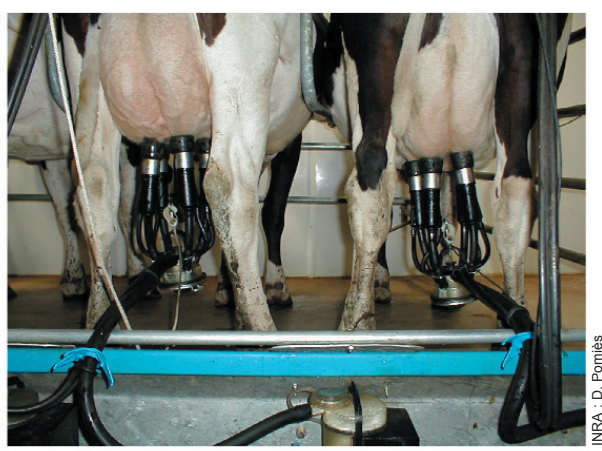

mamelles avant la traite du matin et des vocalisations quand les animaux témoins partent à la traite du soir. Quand la monotraite débute au cours de la phase descendante de la lactation, l'habituation des vaches est encore plus rapide. Au pâturage, seules quelques vaches montrent des signes d'excitation à l'heure de la traite omise (déplacements, vocalisations...), durant moins de $48 \mathrm{~h}$ (Pomiès et al 2007). Néanmoins des études récentes centrées sur le bien-être (Brulé et al 2003, Tucker et al 2007), suggèrent que la pression intra-mammaire occasionnerait une gêne chez les vaches en monotraite en début de lactation.

\section{2 / Variabilité de la baisse de production laitière}

Chez la vache laitière, la monotraite provoque systématiquement une diminution immédiate de la production laitière (figure 1A). Au cours de 13 essais réalisés dans les Unités Expérimentales INRA d'Auvergne (Monts Dore et Marcenat), l'ampleur de la diminution (calculée par rapport à des vaches témoins traites 2 fois par jour) a été de 19 à $46 \%$, selon les lots et les facteurs de variation testés, comparable aux données de la bibliographie (Davis et al 1999). Ces chiffres masquent une sensibilité individuelle à la monotraite encore plus élevée (de moins de 10 à plus de $50 \%$ de perte), sans liaison avec les capacités de production laitière (Rémond et Pomiès 2005) et très répétable au cours de la lactation (Rémond et Pomiès 2007a).

Quand la monotraite est appliquée dès le vêlage, l'écart de production augmente rapidement, jusqu'à dépasser les $30 \%$ après le $1^{\text {er }}$ mois de lactation (figure 1B, Rémond et al 1999). Si la monotraite se prolonge pendant toute la lactation, cet écart (en \%) reste à peu près stable jusqu'au tarissement (figure 1C, Rémond et al 2004). Chez des vaches en monotraite durant plusieurs lactations complètes successives, cet écart ( $>45 \%$ chez les primipares) diminue avec le rang de lactation, notamment entre la 1ère et la 2 ème lactation (Pomiès et Rémond 2007). Quand la monotraite est mise en œuvre après le pic de lactation, la perte de lait n'est en moyenne que de $25 \%$ (figure 1D) et ne dépend pas de la durée de cette période (Rémond et Pomiès 2005). Cet écart peut également varier selon la race, comme par exemple entre vaches Friesian et Jersey (Carruthers et al 1993). Ainsi, à stade de lactation identique, nous avons montré que les vaches Montbéliarde perdent moins de lait que les Holstein (19\% vs 25\%) (Pomiès et al 2007), tandis que dans des conditions proches ce sont les Tarentaise qui perdent le plus (Pomiès et al 2004). Enfin, la réduction des apports d'aliment concentré chez des vaches en monotraite (qui peut paraître justifiée au vu de leur besoins moindres) accroît la perte de lait par rapport à la seule mise en oeuvre de la monotraite, avec un impact proche de celui observé chez des vaches en traite biquotidienne (O'Brien et al 2005, Rémond et al 2005, Guinard-Flament et al 2007a).

Quand les animaux sont à nouveau traits 2 fois par jour, il existe un effet rémanent de la monotraite, qui ne leur permet pas de retrouver le niveau de production des témoins. Cet effet s'accroît avec la durée de monotraite (de 3 à $10 \%$; figures $1 \mathrm{~A}$ et $1 \mathrm{D}$ ) et sa précocité dans la lactation (jusqu'à 16\%, figures $1 \mathrm{D}$ et $1 \mathrm{~B}$ ) (Rémond et Pomiès 2005). Cet effet rémanent n'existe cependant pas d'une lactation à l'autre, même après 3 lactations successives en monotraite (figure 2, Pomiès et Rémond, non publié).

\section{3 / Composition et qualité du lait et des fromages}

La monotraite entraîne des modifications de composition et de qualité du lait qui peuvent avoir une incidence souvent positive sur le prix payé aux éleveurs. Au cours des mêmes essais INRA, la monotraite a provoqué une augmentation moyenne du taux butyreux de $3,0 \mathrm{~g} / \mathrm{kg}(-1,3$ à $+6,0)$, du taux protéique de $1,4 \mathrm{~g} / \mathrm{kg}(-0,2$ à $+3,7)$ et une diminution du taux de lactose de $1,4 \mathrm{~g} / \mathrm{kg}(-0,4$ à $-2,7)$. L'augmentation du taux protéique est due pour $1,0 \mathrm{~g} / \mathrm{L}$ aux caséines et pour $0,8 \mathrm{~g} / \mathrm{L}$ aux protéines solubles, ce qui fait diminuer le rapport «caséines/protéines totales» de 82,0 à $80,8 \%$. Cela ne modifie pourtant pas l'aptitude technologique du lait à la fabrication fromagère (Cantal), ni les caractéristiques principales (composition, rhéologie, sensorielles) des fromages (Pomiès et al 2003). Cependant, cette technique n'est pas encore permise par le cahier des charges de nombreux fromages AOC (St Nectaire fermier, Comté...). La monotraite se traduit aussi par des variations importantes de la teneur en $\operatorname{IgG}(+28 \%)$ et en acides gras libres $(-47 \%)$ du lait. Il n'y a pas d'effet rémanent durable de la monotraite sur la composition du lait après retour à la traite biquotidienne. 
Figure 1. Différence de production laitière entre vaches en monotraite (1T) et en traite biquotidienne (2T), et effet rémanent après retour à 2 traites par jour (encadré grisé), au cours de 4 essais : A) 7 jours de monotraite en phase descendante de lactation ; B) 6 semaines de monotraite dès le vêlage ; C) monotraite durant une lactation complète ;D) 7 semaines de monotraite en phase descendante de lactation.

$1 \mathrm{~A}$

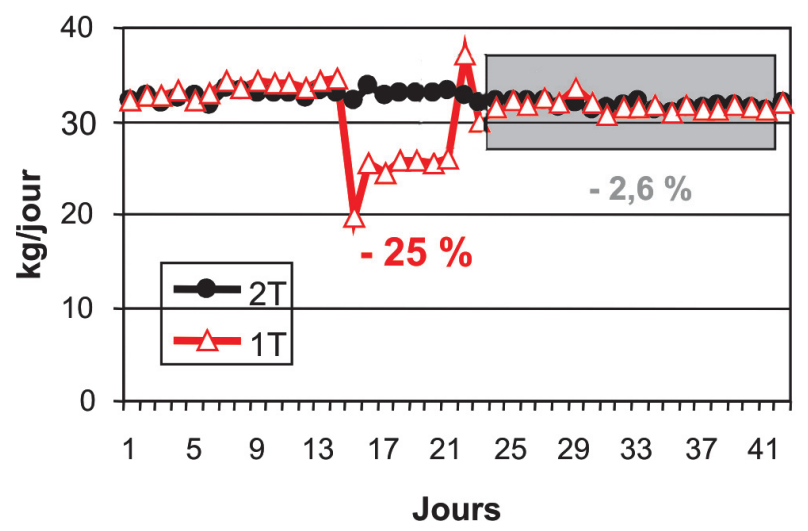

1B
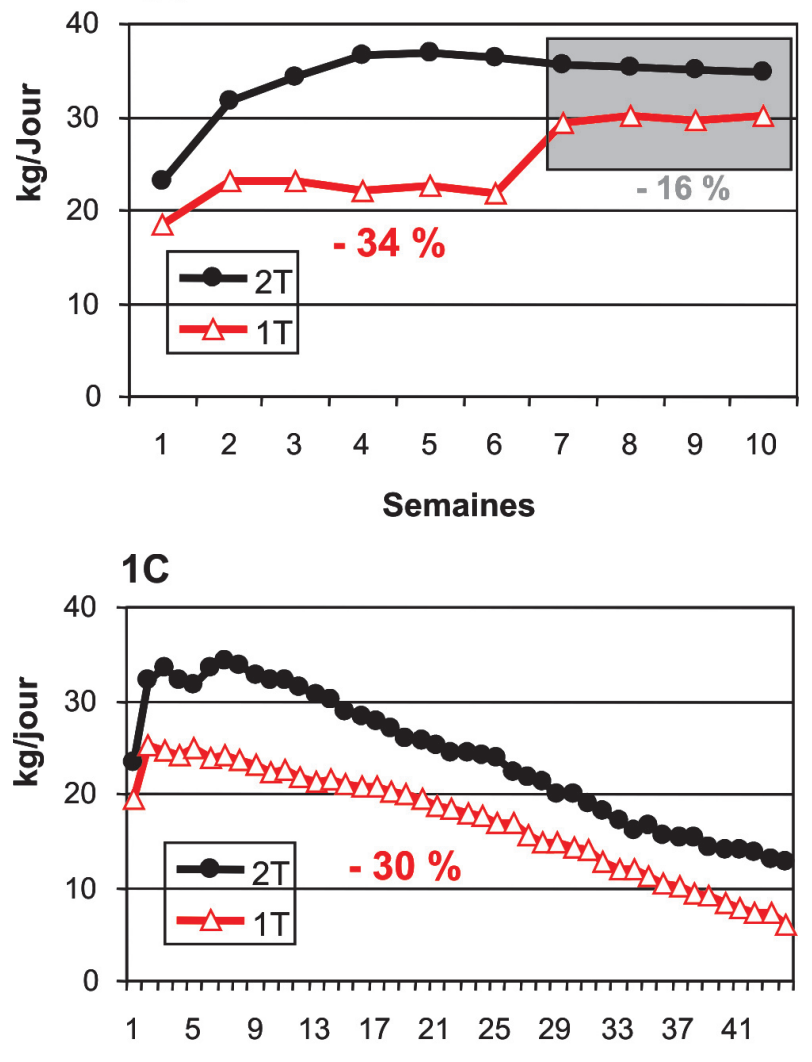

Semaines

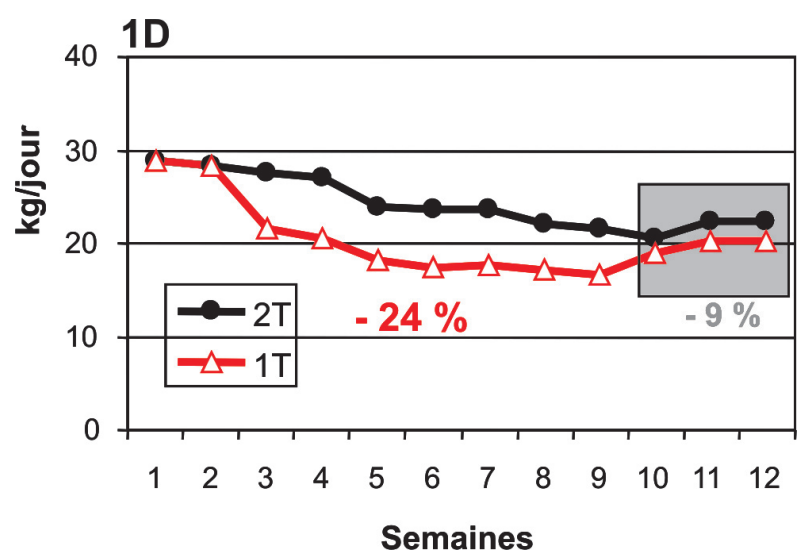

\section{4 / Alimentation, bilan nutri- tionnel et reproduction}

Malgré une production laitière immédiatement plus faible, les vaches en monotraite ont, pendant quelques semaines, une consommation d'aliments identique à celle de vaches en traite biquotidienne alimentées de la même façon («ration complète à volonté» ou «fourrage à volonté + aliment concentré en quantité équivalente»). En début de lactation, cela se traduit par une reprise de poids et d'état corporel plus rapide : la différence avec des animaux témoins vêlant à l'automne avoisine $45 \mathrm{~kg}$ de poids vif et 1 point de note d'état (échelle de 0 à 5) à la mise à l'herbe (Pomiès et Rémond 2002). Ce meilleur bilan nutritionnel des vaches en monotraite est confirmé par des teneurs plasmatiques plus élevées en glucose et plus faibles en acides gras non estérifiés (Holmes et al 1992, Rémond et al 1999, 2005). Une conséquence de ce bon bilan (liée à la corrélation entre état nutritionnel et reprise de la cyclicité ovarienne après vêlage) est une détection des chaleurs souvent plus précoce chez les vaches en monotraite (Patton et al 2006, Rémond et Pomiès 2007b) et une diminution de l'intervalle vêlage-insémination fécondante (- 24 jours chez des primipares, $\mathrm{p}<0,05$, Pomiès et Rémond non publié).

\section{5 / Numération cellulaire et pathologie mammaire}

En phase descendante de lactation, le passage en monotraite provoque une augmentation rapide, importante (x 3) mais transitoire (4-5 jours) de la concentration en cellules somatiques du lait (Rémond et Pomiès 2007b). Au-delà, et jusqu'à 10 semaines de monotraite, une faible différence (environ +30000 cellules $/ \mathrm{mL}$ ) persiste avec les témoins, sans que cette augmentation soit associée à la présence de mammites cliniques. Quand la monotraite est mise en œuvre dès le vêlage et se poursuit durant une grande partie, voire toute la lactation, la concentration en cellules somatiques s'accroît plus rapidement que chez les animaux témoins, jusqu'à atteindre des niveaux pénalisants pour le paiement du lait aux éleveurs (Rémond et al 2004). De plus, cette pratique entraîne, au moins chez les primipares, un nombre de mammites cliniques significativement plus élevé (Pomiès et Rémond non publié). 
Figure 2. Evolution hebdomadaire de la production laitière de 74 vaches Holstein primipares en traite biquotidienne (2T) ou en monotraite (1T) durant 3 lactations successives, avant un régime de traite commun (2 fois par jour) en 4ème lactation.

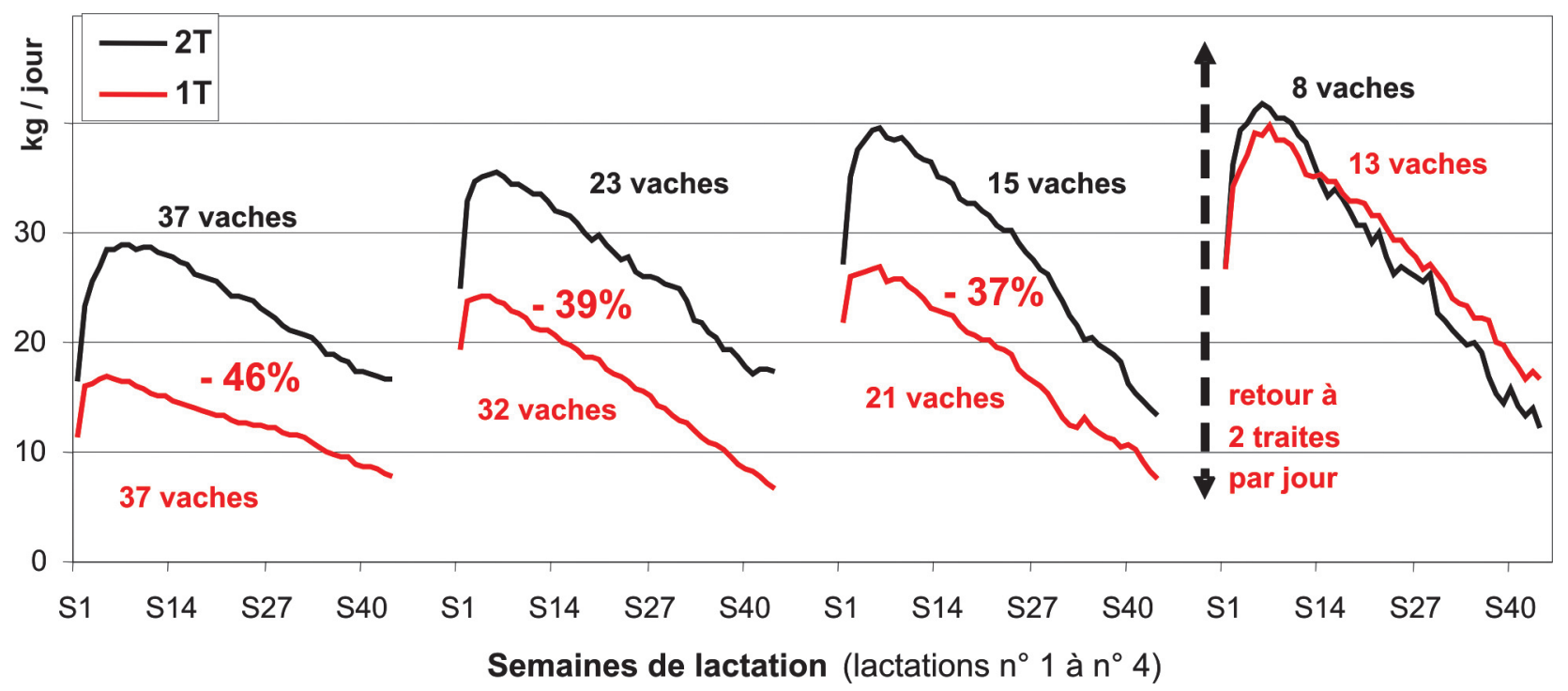

\section{6 / Economie de l'élevage}

Il existe très peu d'études ou d'enquêtes sur l'impact économique de la monotraite dans les élevages bovins laitiers. Deux simulations ont été réalisées par l'ENITAC à partir de données réelles recueillies dans des exploitations en traite biquotidienne, auxquelles ont été appliqués les résultats zootechniques issus des essais de l'INRA sur la monotraite (Brunschwig et al 2003, 2004). Il en ressort que la monotraite utilisée entre 1 semaine et 3 mois durant l'été (à quota constant) permettrait d'augmenter le temps disponible calculé (annuel) de 0,6 à 6,8\% malgré un léger accroissement du cheptel $(<5 \%)$, mais avec une diminution de l'Excédent Brut d'Exploitation (EBE) de 0,1 à $3,1 \%$. Par contre, utilisée toute l'année, la monotraite obligerait à accroître le cheptel de $39 \%$ pour que la production de lait soit maintenue, et de ce fait, elle ne permettrait pas vraiment une diminution de la charge de travail. A cheptel constant, le revenu de l'éleveur serait fortement touché, avec une diminution moyenne de $62 \%$ de l'EBE. Une autre simulation sur l'utilisation de la monotraite toute l'année, réalisée par la Chambre d'Agriculture du Finistère et l'Institut de l'Elevage (Portier et al 2006), montre néanmoins que sous certaines conditions la marge brute d'exploitation peut être identique, avec un accroissement de $27 \%$ du cheptel et une diminution de $17 \%$ du travail d'astreinte.

\section{7 / Cas des petits ruminants}

A l'INRA, les études de monotraite en petits ruminants sont bien plus anciennes, que ce soit chez la brebis (Casu et Labussière 1972) ou chez la chèvre (Mocquot 1980). Cependant, il existe peu de travaux récents pour évaluer la réponse zootechnique des petits ruminants à la monotraite (Salama et al 2003), et ceux plus anciens ou sur de courtes périodes pour des études physiologiques (Boutinaud et al 2003a, Negrão et al 2001) donnent des pertes de lait très variables, s'échelonnant de 5 à $41 \%$. De fait, comme pour les vaches, les éleveurs ont des craintes quant à l'impact d'une telle conduite chez des animaux fort producteurs. L'équipe de l'INRA de Rennes, en collaboration avec la ferme expérimentale du Pradel (PEP caprin Rhône-alpes), teste donc cette conduite depuis 5 ans chez des chèvres de race Alpine (850 à $1100 \mathrm{~kg}$ de lait par lactation) en monotraite dès le vêlage (au Pradel) ou après environ un mois de lactation (Marnet et al 2005).

Les chèvres en monotraite ne présentent pas de trouble du comportement (vocalisations, agitation) ni d'écoulement de lait de la mamelle, et aucune mammite clinique ne s'est déclarée au cours des 3 premières années. Les chèvres perdent en moyenne $15 \%$ de lait, que la monotraite soit appliquée en première ou seconde partie de lactation, avec cependant une très forte variabilité individuelle (-2 à - 30\%). Cette variabilité est encourageante pour une sélection future des animaux les plus aptes à tolérer de grands intervalles de traite. L'application de la monotraite dès la mise bas semble en revanche plus difficile à supporter pour les primipares, qui perdent plus de $24 \%$ de lait, suggérant qu'un développement mam- maire optimal est requis pour minimiser l'impact de cette technique. Chez la chèvre, le taux butyreux n'augmente que très faiblement en monotraite $(+0,8 \mathrm{~g} / \mathrm{L})$, tandis que le taux protéique s'accroît significativement $(+2,7 \mathrm{~g} / \mathrm{L})$, principalement en seconde moitié de lactation. Mais, comme chez la vache, la lipolyse est réduite d'environ $50 \%$ lors d'intervalles de traite de $24 \mathrm{~h}$. Un accroissement du nombre de chèvres présentant une «inversion de taux» (taux butyreux $<$ taux protéique) en milieu de lactation a également été observé certaines années, suggérant la nécessité de réduire l'apport d'aliment concentré chez les animaux en monotraite. L'intégrité de l'épithélium sécréteur mammaire est globalement maintenue, avec un rapport $\mathrm{Na}^{+} / \mathrm{K}^{+}$non modifié dans le lait. Seule une légère mais significative augmentation de la concentration en albumine sérique mesurée dans le lait après le changement de rythme de traite $(+221 \mu \mathrm{g} / \mathrm{mL}$ au 4ème jour) suggère une fuite transitoire de protéines solubles sanguines, sans effet sur le rapport "caséines/protéines solubles» du lait. De même, malgré l'augmentation du taux protéique, les rendements fromagers réels (AOC Picodon) ne semblent pas significativement meilleurs avec la monotraite. La concentration en cellules somatiques n'augmente pas significativement, suggérant une faible inflammation du tissu mammaire. Il faut cependant noter ici le niveau particulièrement bas en cellules somatiques du troupeau expérimental, qui ne permet pas d'extrapoler à ce qui se passerait en élevage avec un niveau supérieur. Par ailleurs, les mamelles n'ont pas été altérées par la répétition du traitement sur plusieurs 
lactations. La persistance laitière semble même meilleure et aucun effet rémanent sur le potentiel des animaux n'a été observé au cours de la lactation suivante.

La monotraite, apparemment mieux supportée par la chèvre que par la vache, apparait donc comme une alternative avantageuse pour une simplification du travail chez cette espèce.

\section{8 / Approche mécaniste}

Les études mécanistes menées à l'INRA sur la monotraite s'inscrivent dans le cadre plus général de travaux qui visent à comprendre comment la fonction mammaire est affectée par des variations de la fréquence de traite, ou de la qualité de la vidange mammaire.

Les travaux ayant pour but d'identifier et de hiérarchiser les signaux générant la perte de lait montrent l'importance des phénomènes locaux (liés à l'accumulation du lait dans la mamelle) par rapport aux régulations d'origine systémique (liées aux hormones relarguées pendant et entre les traites). Les théories de régulation locale physique et chimique coexistent sans pour autant que l'identification d'un facteur chimique présent dans le lait et capable d'exercer un rétro-contrôle négatif sur sa propre synthèse n'ait à ce jour abouti chez la vache et la brebis. Les approches nutritionnelles ont permis d'étudier comment l'utilisation des nutriments, précurseurs des composants du lait, est modifiée au niveau de la mamelle en réponse à la monotraite (Guinard-Flament et al 2006). Elles montrent l'importance du débit sanguin mammaire, qui chute de 10 à $17 \%$ chez les vaches en monotraite, dans la réduction du flux de nutriments arrivant à la mamelle. Le métabolisme intra-mammaire est lui aussi modifié : diminution des synthèses et, dans certains cas, réorientation des précurseurs énergétiques vers la synthèse des matières grasses (Delamaire et Guinard-Flament 2006a, 2006b). La persistance de la lactation pourrait, quant à elle, être modifiée via des variations du nombre et de l'activité des cellules sécrétrices (Boutinaud et al 2003b) et de la réceptivité du tissu mammaire aux hormones galactopoiétiques. Les travaux sur les facteurs déterminant l'aptitude des animaux à supporter la monotraite montrent l'importance de la capacité de stockage de la mamelle et de la répartition du lait dans la glande entre les traites sur l'inhibition de la synthèse du lait. La taille de la citerne, la compliance de la mamelle (capacité à se dilater) et les modalités de transfert du lait dans la mamelle entre les traites apparaissent comme des facteurs clés, sans que leur importance relative soit connue pour chacune des espèces. La taille de la citerne joue un rôle essentiel chez la vache et la brebis, mais ne serait pas limitante chez la chèvre (Marnet et Komara 2008). Les travaux consistant à rechercher des indicateurs synthétiques capables de prédire la sensibilité des animaux à la monotraite constituent un enjeu fort pour le développement de cette pratique. Ainsi, les premiers résultats obtenus sur le lactose plasmatique chez la vache restent à confirmer (Guinard-Flament et al 2007b).

Pour réduire l'astreinte de la traite durant quelques semaines en dehors de la phase ascendante de lactation, la monotraite est une pratique sans risque, facile et rapide à mettre en œuvre, et complètement réversible. Mais chez les bovins, utilisée de façon prolongée dès le vêlage, elle pénalise fortement la lactation en cours et peut présenter un risque sanitaire pour l'animal.

\section{4 / Les autres voies de recherche}

Le robot et la monotraite ne sont pas les seules solutions sur lesquelles l'INRA a travaillé ces 20 dernières années pour alléger l'astreinte biquotidienne de la traite chez les ruminants.

\section{1 / La suppression de la traite du dimanche soir}

Cette conduite a été pour la première fois étudiée en France dans les années 60-70, chez la vache (Labussière et Coindet 1968) et la brebis (Labussière et al 1974), sans trouver beaucoup d'écho : la production laitière n'était alors pas limitée et la recherche de temps libre n'était pas une priorité des éleveurs. De plus, les pertes de lait enregistrées étaient assez fortes : 7 à $10 \%$ chez la vache laitière; près de $25 \%$ chez la brebis Préalpes du Sud ; $13 \%$ chez la brebis Sarde, race forte productrice à grande citerne. Elle a retrouvé un regain d'intérêt dans les années 90 auprès des élevages familiaux de vaches laitières, sans main-d'œuvre externe. Selon une enquête du Contrôle Laitier, près de $17 \%$ des éleveurs du Finistère l'appliquerait (Portier et al 2006), ce qui en fait la technique ayant le plus fort taux de pénétration dans les élevages.
A l'initiative de la filière laitière, craignant de fortes variations de la qualité technologique des laits, un essai a été réalisé en 2001 et 2002 par l'INRA de Rennes et l'Institut de l'Elevage (Meffe et al 2003), afin de réévaluer cette pratique sur des vaches Holstein fortes productrices $(>9000 \mathrm{~kg}$ de lait par lactation). Comme chez de nombreux éleveurs, la traite du dimanche matin était retardée de $3 \mathrm{~h}$ afin de limiter à $21 \mathrm{~h}$ l'intervalle avec celle du lundi matin.

Les vaches se sont vite adaptées à ce rythme, malgré des vocalisations au moment de la traite omise durant 2 à 4 week-ends et des écoulements de lait sur la litière chez une bonne partie des animaux durant le premier tiers de la lactation. La perte de lait estimée (en prenant la fin de semaine comme référence de retour à la normale) est voisine de $3 \%$, avec une forte augmentation du taux butyreux le lundi soir $(+6 \mathrm{~g} / \mathrm{kg})$ mais sans modification $\mathrm{du}$ taux protéique. La concentration en cellules somatiques augmente fortement le lundi soir, jusqu'à un retour progressif à la normale le mercredi soir (soit $+17 \%$ en moyenne sur la semaine), sans s'accompagner d'une incidence supérieure de mammite clinique dans un troupeau sain. La qualité fine du lait ( $\mathrm{pH}$, point de congélation, concentrations en calcium, chlorures et phosphore, lipolyse, profils d'acides gras, rapport caséines/protéines solubles, rapports entre les différentes caséines, urée, lactopéroxydase, IgG, BSA, $\beta$ lactoglobuline, $\alpha$ lactalbumine) n'a pas été modifiée par cette conduite, ce qui a permis de rassurer les transformateurs sur ses aptitudes technologiques.

Quand l'horaire de la traite du dimanche matin n'est pas modifié (intervalle de $24 \mathrm{~h}$ ), les pertes de lait sont plus importantes (- 10\%) mais les répercussions sur la composition du lait sont identiques (figure 3, Pomiès et Rémond 2000).

Cette conduite, bien tolérée par l'animal et la glande mammaire y compris chez des animaux forts producteurs, peut donc être recommandée sur toute ou partie de la lactation, sous réserve d'une bonne santé de la mamelle et d'une résistance suffisante des sphincters des trayons.

\section{2 / Trois traites en deux jours}

Cette pratique n'est pas utilisée couramment car elle impose trois horaires de traite différents, avec souvent une traite nocturne. Elle a été testée en 1995 
Figure 3. Evolution hebdomadaire traite par traite de la production laitière $(P L)$, des taux butyreux (TB) et protéique (TP), et de la concentration en cellules somatiques (CCS) du lait, chez des vaches traites matin et soir toute la semaine (témoin) et chez des vaches dont la traite du dimanche soir est supprimée (13 traites).
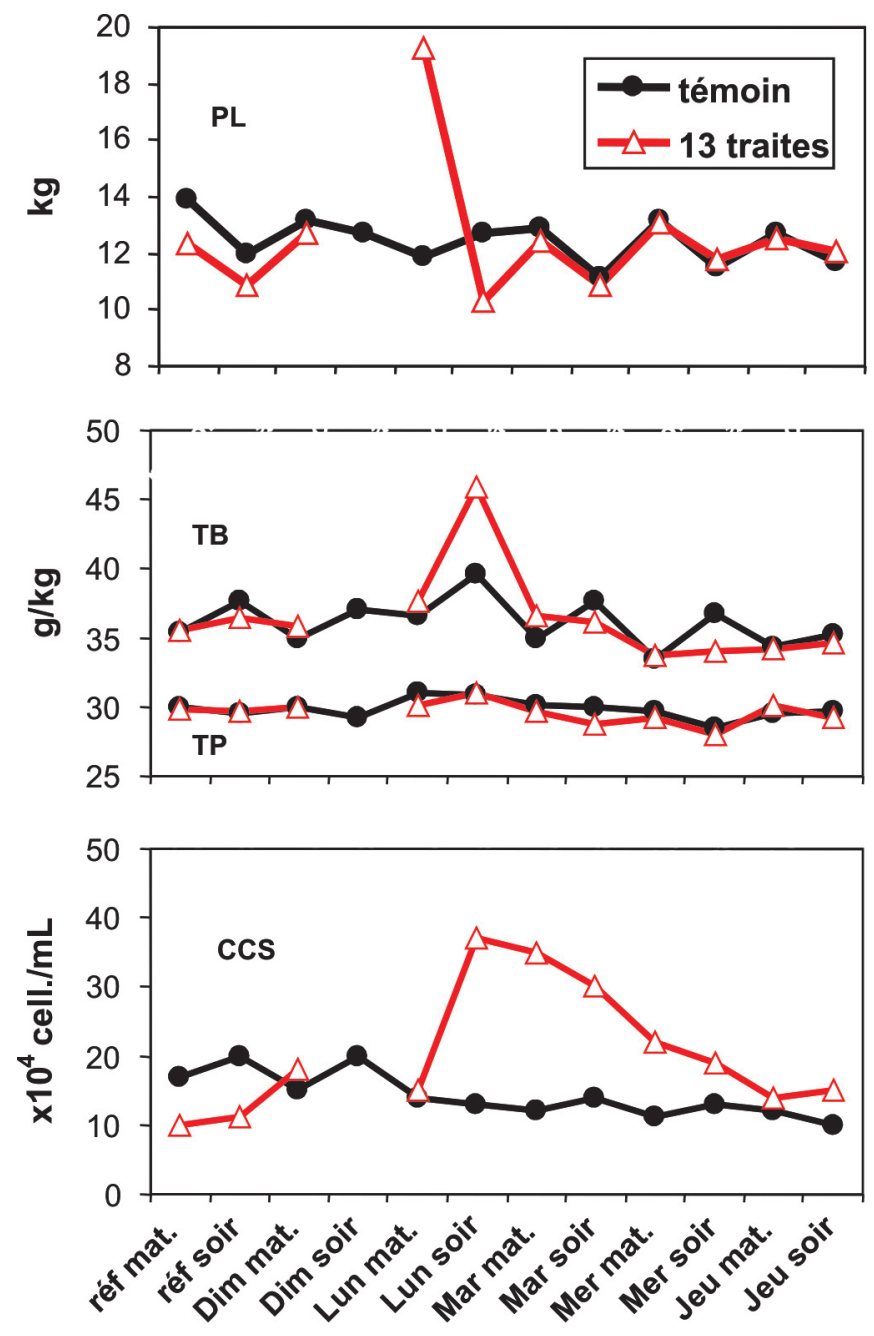

à l'INRA, chez la vache laitière, avec des horaires irréguliers $(6 \mathrm{~h}, 20 \mathrm{~h}$ et $14 \mathrm{~h}$ le lendemain) (Rémond et Boit 1997). En début de lactation, cette pratique n'a pas entraîné de modifications significatives chez les multipares, mais une diminution de $16 \%$ du lait produit par les primipares, accompagnée d'une augmentation des taux butyreux et protéique. En phase descendante de lactation, la production de lait a diminué de $10 \%$ sans modification de composition.

Un rythme de traite régulier de $16 \mathrm{~h}$ chez des brebis laitières entre le 90ème et le 160 ème jour de lactation a été expérimenté à l'Université du Wisconsin, en collaboration avec l'INRA de Rennes. Cela n'a pas entraîné de différence significative de durée de lactation, de production de lait, de concentrations en matières grasse, protéique et en cellules somatiques (Mckusick et al 2002a), tandis que le temps de traite était réduit de $27 \%$. les Unités Expérimentales d'Auvergne (Rémond et al 2006) ont permis de montrer que sur une courte période en phase descendante de lactation, un intervalle de 3 ou $5 \mathrm{~h}$ entre les 2 traites d'une même journée ne réduisait la production laitière que de $11 \%$ et $3 \%$ respectivement, un intervalle de $7 \mathrm{~h}$ n'ayant même aucune incidence. La mise en place dès le vêlage et durant la moitié de la lactation de 2 traites dans la matinée (à $6 \mathrm{~h}$ et $11 \mathrm{~h}$ ) entraîne une diminution de la production laitière de $10 \%$ et une augmentation équivalente du taux butyreux, sans conséquence sur la numération cellulaire et sans effet rémanent quand l'intervalle entre les 2 traites repasse à $10 \mathrm{~h}$.

Cette conduite, qui ne permet pas de réduire la charge de travail mais qui affecte peu la production laitière, peut se révéler utile pour des éleveurs souhaitant dégager du temps les aprèsmidi.

\section{4 / La monotraite en présence du jeune}

Cette conduite mixte (une seule traite + tétée libre des jeunes une partie de la journée) n'est pas utilisée en vache et chèvre laitières, malgré un intérêt grandissant des éleveurs engagés dans des systèmes d'agriculture biologique ou de fabrication fromagère à la ferme. C'est en revanche une pratique courante des éleveurs de brebis laitières qui s'est imposée dans les années 80 , avec l'augmentation du potentiel laitier des brebis sous l'effet de la sélection, telle en race Lacaune (Barillet 1989). Traditionnellement autour de la Méditerranée, le premier mois de lactation des brebis, voire des chèvres, est consacré à l'allaitement des agneaux. La brebis est donc d'abord exploitée comme une femelle allaitante pour produire des agneaux de lait élevés sous la mère, puis après le sevrage à un mois comme une femelle traite pour valoriser le lait en fromage. Mais l'accroissement du potentiel laitier a imposé de traire, une fois par jour pendant ce premier mois d'allaitement, le lait excédentaire non tété par les agneaux. Cette conduite s'est donc développée pour maintenir la production d'agneaux de lait sous la mère (très bien valorisée), sans freiner la lactation des brebis fortes productrices dont les petits ne peuvent à eux seuls vider totalement la mamelle. Une telle pratique évite de recourir à l'allaitement artificiel systématique des agneaux, qui constitue un chantier prohibitif en travail étant donné la taille des troupeaux qu'il 
Photo 3. Vaches en monotraite avec leurs veaux dans la stabulation de l'Unité Expérimentale des Monts Dore.

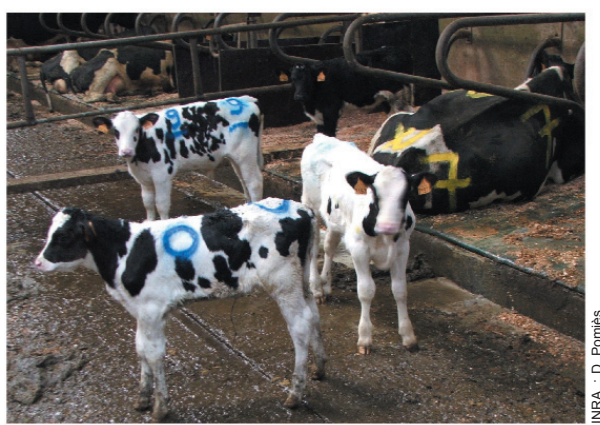

s'agit de traire par ailleurs. Enfin, les éleveurs pensaient qu'une telle conduite permettait d'habituer les animaux à venir en salle de traite afin de limiter les problèmes d'adaptation après le sevrage. Après avoir validé la sélection laitière dans un tel système (Barillet 1989) et vérifié le niveau du pic de lactation atteint durant ce premier mois d'allaitement + traite (Bocquier et al 1999), nous avons montré que cette technique ne permettait pas de mieux adapter les animaux à la traite mécanique après sevrage. En effet, au plan physiologique, les travaux de l'INRA ont mis en évidence que l'existence d'un lien mère-jeune et d'un comportement maternel bloquait la décharge d'ocytocine à la traite sans affecter celle de prolactine et de cortisol chez la brebis et la chèvre (Hernandez et al 2002, Marnet et Negrão 2000), ni le taux de GH chez la vache (De Passillé et al 2007). De fait, malgré une éjection de lait incomplète à la traite, mais grâce aux décharges plus nombreuses des autres hormones galactopoïetiques ainsi qu'à la baisse de la pression intramammaire, la lactation n'est pas freinée et peu même être augmentée significativement par rapport à une conduite à deux traites exclusives par jour. Un essai conduit sur des brebis frisonnes à Spooner durant les 6 premières semaines de lactation (Mckusick et al 2002b) a montré que cette conduite mixte bloque bien l'éjection du lait et réduit la quantité récoltée à la traite de 40 à $60 \%$. En revanche la production de lait totale (traite + tétée) est augmentée de $42 \%$, démontrant l'effet galactopoïétique des tétées additionnelles. Le taux protéique du lait trait reste inchangé, le taux butyreux diminue suite à la rétention de la matière grasse dans le lait alvéolaire, et aucun effet significatif n'est observé sur la concentration en cellules somatiques, suggérant une pratique respectueuse de la physiologie mammaire. Nos travaux récents (Hernandez et al 2007) montrent également que cette conduite pourrait s'appliquer dans les élevages familiaux des pays du sud, où il faut souvent choisir entre se nourrir du lait des chèvres et élever les cabris.

Cette technique, respectueuse de la séquence comportementale maternelle de l'animal et qui permet à l'éleveur de réduire son travail, est actuellement expérimentée à l'INRA chez des vaches laitières hautes productrices (Pomiès et Rémond, non publié). Elle reste cependant fortement déconseillée chez les éleveurs de petits ruminants qui cherchent à éradiquer les infections rétrovirales de type CAEV (Caprine Arthritis Encephalitis Virus) ou MVV (Maedi Visna Virus), pour lesquelles la tétée est une des voies majeures de contamination des jeunes.

\section{5 / Vers une sélection sur la forme de la courbe de lactation et l'aptitude à la monotraite}

Un projet pluridisciplinaire concernant l'expression et l'entretien de la lactation des brebis selon la persistance laitière et le nombre de traites par jour a débuté au domaine INRA de La Fage en 2007. Le recours à la monotraite, actuellement interdite dans l'AOC Roquefort, a supposé une demande d'autorisation expérimentale auprès de l'Interprofession du Bassin, devenue ainsi partenaire du projet. Celui-ci est fondé sur un dispositif de lignées Lacaune divergentes pour la persistance laitière, subissant une ou deux traites par jour. Il s'agit, avec une entrée génétique sur la persistance laitière, de tester l'impact de divers systèmes d'élevage innovants (monotraite, alimentation selon le pic de lactation ou non) sur l'entretien de la lactation, les qualités chimiques, hygiéniques et nutritionnelles des laits, la facilité de traite à la machine, la santé de la mamelle et le bien-être animal, l'état nutritionnel des brebis, l'étalement de la production recherché par l'aval, le temps de travail à la traite et les coûts de production. Nous faisons en particulier l'hypothèse que le robot de traite ne se développera pas en petits ruminants et que la monotraite, si elle est bénéfique, constituera l'ajustement majeur possible de la productivité du travail à la traite pour ces filières. L'un des objectifs génétiques concrets de ce programme est de déterminer si les brebis à courbe de lactation «plus plate» supportent mieux la monotraite. L'autre objectif génétique vise à identifier les principaux caractères qui sont impliqués dans l'aptitude à maintenir la lactation, quel que soit le nombre de traites journalières. On pourra ainsi vérifier s'ils sont, au moins partiellement, pris en compte dans les caractères fonctionnels récemment intégrés dans les nouveaux objectifs de sélection des petits ruminants laitiers (Barillet 2007), ou valider s'il convient de favoriser leur sélection. De façon plus générale, ce projet est considéré comme générique en génétique pour les espèces laitières, la brebis constituant un modèle pertinent pour la vache du point de vue des simplifications touchant à la traite.

\section{Conclusion}

De nombreuses conduites de la traite, alternatives à la traite «classique» 2 fois par jour, existent et permettent de répondre en partie aux aspirations fortes des éleveurs à une meilleure qualité de vie. La pénétration de ces conduites dans les élevages, qui est encore globalement très limitée, dépendra de nombreux facteurs dont il est toujours risqué d'anticiper le poids. On peut toutefois noter que : 1/ la traite robotisée est la seule conduite qui ne diminue pas la production laitière puisqu'elle permet, sans effort, d'accroître la fréquence de traite. Elle permet donc à l'éleveur de mieux répondre à la récente et brusque demande d'augmentation de la production laitière bovine. Elle a cependant contre elle un coût (encore ?) élevé par litre de lait, une modification de la conduite d'élevage allant vers plus de stabulation des animaux, et d'être peu réversible ; 2/ toutes les autres conduites sont fondées sur l'allongement plus ou moins important (jusqu'à $24 \mathrm{~h}$ ) et/ou plus ou moins fréquent de l'intervalle entre (certaines) traites, par rapport à l'intervalle de traite «classique» de $12 \mathrm{~h}$. Ces conduites entraînent donc toutes une diminution plus ou moins sensible des performances individuelles des animaux et certaines résisteront peut-être mal aux accroissements de la demande de lait et de son prix, s'ils durent, ainsi qu'à la suppression annoncée des quotas laitiers. De plus, l'allongement de l'intervalle de traite à $24 \mathrm{~h}$ en phase ascendante de la lactation, même quand il est irrégulier (omission de la traite du dimanche soir), semble pouvoir entraîner une "gêne» chez la vache qu'il importe de mieux préciser.

Ces inconvénients sont entièrement liés à la capacité limitée des femelles laitières à tolérer de longs intervalles entre les traites. Or, cette tolérance présente de grandes variations, non seulement entre les espèces (les chèvres 
étant plus tolérantes que les vaches) mais aussi entre les individus. Le développement de ces conduites repose donc certainement en bonne partie sur la possibilité d'identifier les déterminants génétiques de cette aptitude et de les sélectionner.

\section{Remerciements}

Les auteurs tiennent à remercier l'ensemble du personnel INRA des Unités Expérimentales des Monts Dore, de Marcenat, du Pin, de La Fage, et de l'Installation Expérimentale de l'UMRPL de Rennes pour leur contribution indispensable à la mise au point et à l'évaluation de ces conduites de traite innovantes.

\section{Références}

Barillet F., 1989. Expression de la production laitière à la traite des brebis Lacaune en système allaitement $\mathrm{x}$ traite mécanique. 4 ème Symp. Int. sur la traite des petits ruminants, Tel-Aviv, Israël, 13-19 septembre, 463-495.

Barillet F., 2007. Genetic improvement for dairy production in sheep and goats. Small Rum. Res., 70, 60-75.

Barthez A., 1986. Du labeur paysan au métier d'agriculteur : l'élaboration statistique en agriculture. Cahiers Econ. Sociol. Rur., 45-72.

Billon P., Pomiès D., 2006. Le point sur la robotisation de la traite 15 ans après l'apparition des premiers systèmes dans les fermes. Renc. Rech. Rum., 13, 143-150.

Bocquier F., Aurel M.R., Barillet F., Jacquin M., Lagriffoul G., Marie C., 1999. Effects of partial-milking during the suckling period on milk production of Lacaune dairy ewes. In : Milking and milk production of dairy sheep and goats. Proc. $6^{\text {th }}$ Int. Symp. milking of small Ruminants, Athens, Greece, 26 September-1 October 1998, 257-262.

Bony J., 2002. Comportement des vaches au robot. In : Le robot de traite : aspects techniques et économiques. Bony J., Pomiès D. (Eds), INRA Editions, Paris, France, 50-52.

Boutinaud M., Guinard-Flament J., Jammes H., 2003a. Analyse de l'expression de gènes indicateurs de la mort cellulaire dans la glande mammaire, un indice du potentiel de production de la glande mammaire. Renc. Rech. Rum., 10, 49-52.

Boutinaud M., Rousseau C., Keisler D.H., Jammes H., 2003b. Growth hormone and milking frequency act differently on goat mammary gland in late lactation. J. Dairy Sci., 86, 509-520.

Brulé A., Brocard V., Portier B., Racine V., 2003. Effets de la réduction de la fréquence de traite sur le bien-être de la vache laitière. Renc. Rech. Rum., 10, 77-80.

Brunschwig G., Rémond B., Pomiès D., Martin B., Vidal L., 2003. Simulation de la traite des vaches laitières une seule fois par jour en période estivale ou durant toute l'année : effets sur le revenu et le temps dégagé au niveau de l'exploitation. Renc. Rech. Rum., 10, 100.

Brunschwig G., Babault S., Rémond B., Pomiès D., 2004. Simulation de la traite des vaches laitières une seule fois par jour durant de courtes périodes (1 à 13 semaines) : effets sur l'EBE et le temps dégagé au niveau de l'exploitation. Renc. Rech. Rum., 11, 231.

Carruthers V.R., Davis S.R., Bryant A.M., Henderson H.V., Morris C.A., Copeman P.J.A., 1993. Response of Jersey and Friesian cows to once a day milking and prediction of response based on udder characteristics and milk composition. J. Dairy Res., 60, 1-11.
Casu S., Labussière J., 1972. Premiers résultats concernant la suppression d'une ou plusieurs traites par semaine chez la brebis Sarde. Ann. Zootech., 21, 223-232.

Chauvat S., Seegers J., N'Guyen The B. Clément B., 2003. Le travail d'astreinte en élevage bovin laitier. Institut de l'Elevage, Paris, France, 50p.

Cournut S., Dedieu B., 2005. Simplification des conduites d'élevage en bovins laitiers. Cahiers Agric., 14, 541-547.

Davis S.R., Farr V.C., Stelwagen K., 1999 Regulation of yield loss and milk composition during once-daily milking: a review. Liv. Prod. Sci., 59, 77-94.

De Passillé A.M., Marnet P.G., Lapierre H., Rushen J., 2008. Effects of nursing a calf on milk ejection and milk yield during nursing and milking. J. Dairy Sci., sous presse.

Dedieu B., Servière G., 2004. Des pistes pour améliorer le travail des éleveurs. Revue POUR $182,32-38$

Dedieu B., Servière G., Madelrieux S., Dobremez L., Cournut S., 2006. Comment appréhender conjointement les changements techniques et les changements du travail en élevage? Cahiers Agric., 15, 506-513.

Delamaire E., Guinard-Flament J., 2006a. Increasing milking intervals decreases the mammary blood flow and mammary uptake of nutrients in dairy cows. J. Dairy Sci., 89, 34393446.

Delamaire E., Guinard-Flament J., 2006b. Longer milking intervals alter mammary epithelial permeability and the udder's ability to extract nutrients. J. Dairy Sci., 89, 2007-2016.

Dufour A., Hostiou N., Cournut S., Dedieu B. 2007. Le travail en élevage laitier : des conceptions, des noyaux organisateurs et des profils d'organisation variés. Renc. Rech. Rum., 14, 385-388.

Guillaumin A., Kling-Eveillard F., Moreau J.C., Marty M., 2005. Résultats d'enquêtes en Aquitaine. Quand les éleveurs laitiers parlent de leurs conditions de travail. Travaux et Innovations, 115, 30-34.

Guinard-Flament J., Delamaire E., Lemosque S., Boutinaud M., David Y., 2006. Changes in mammary uptake and metabolic fate of glucose with once-daily milking and feed restriction in dairy cows. Reprod., Nutr., Dev., 46, 589-598.

Guinard-Flament J., Delamaire E., Lamberton P., Peyraud J.L., 2007a. Adaptations of mammary uptake and nutrient use to once-daily milking and feed restriction in dairy cows. J. Dairy Sci., 90, 5062-5072.

Guinard-Flament J., Gallard Y., Larroque H., 2007b. Le taux de lactose plasmatique, un indicateur de la perte de lait lors du passage en monotraite chez la vache laitière ? Renc. Rech. Rum., 14, 425 .

Hernandez H., Serafin N., Terrazas A.M. Marnet P.G., Kann G., Delgadillo J.A., Poindron P., 2002. Maternal olfaction differentially modulates oxytocin and prolactin release during suckling in goats. Horm. Behav., 42, 232-244.

Hernandez H., Delgadillo J.A., Flores J.A., Rodriguez A.D., Serafin N., Kann G., Marnet P.G., Poindron P., 2007. Effects of restricted nursing on milk production and collection, kid growth and plasma prolactin and growth hormone concentrations in dairy goats. Animal, 1, 233-240.

Holmes C.W., Wilson G.F., Mackenzie D.D.S., Purchas J., 1992. The effects of milking once daily throughout lactation on the performance of dairy cows grazing on pasture. Proc. $\mathrm{N}$ Z. Soc. Anim. Prod., 52, 13-16.

Labussière J., Coindet J., 1968. Effets de la suppression de la traite du dimanche soir, chez les bovins de race Française Frisonne Pie Noire. Ann. Zootechn., 17, 231-244.

Labussière J., Combaud J.F., Petrequin P., 1974. Effets de la suppression de la traite du dimanche soir sur les brebis de race Préalpes du sud. Ann. Zootechn., 23, 435-444.

Marnet P.G., 2002. Réponses et adaptations physiologiques des vaches à la traite robotisée In : Le robot de traite : aspects techniques et économiques. Bony J., Pomiès D. (Eds), INRA Editions, Paris, France, 69-78.

Marnet P.G., Komara M., 2008. Management systems with extended milking intervals in ruminants: regulation of production and quality of milk. J. Dairy Sci., sous presse.

Marnet P.G., Negrão J.A., 2000. The effect of a mixed-management system on the release of oxytocin, prolactin, and cortisol in ewes during suckling and machine milking. Reprod. Nutr. Dev., 40, 271-281.

Marnet P.G., Gomis B., Guinard-Flament J., Boutinaud M., Lollivier V., 2005. Effet d'une seule traite par jour (monotraite) sur les performances zootechniques et les caractéristiques physicochimiques du lait chez les chèvres Alpines à haut potentiel. Renc. Rech. Rum., 12, 225-228.

Mckusick B.C., Thomas D.L., Berger Y.M. Marnet P.G., 2002a. Effect of milking interval on alveolar versus cisternal milk accumulation and milk production and composition in dairy ewes. J. Dairy Sci., 85, 2197-2206.

Mckusick B.C., Thomas D.L., Romero J.E., Marnet P.G., 2002b. Effect of weaning system on milk composition and distribution of milk fat within the udder of East Friesian dairy ewes. J. Dairy Sci., 85, 2521-2528.

Meffe N., Tache C., Gaudin V., Marnet P.G., 2003. Suppression d'une traite par semaine pen- 
dant toute la lactation chez les vaches laitières à haut potentiel : effets zootechniques et caractéristiques physico-chimiques du lait. Renc. Rech. Rum. 10, 85-88.

Mocquot J.C., 1980. La réduction du nombre de traites : mythe ou réalité ? Chèvre, 25-31.

Negrão J.A., Marnet P.G., Labussière J., 2001. Effect of milking frequency on oxytocin release and milk production in dairy ewes. Small Rum. Res., 39, 181-187.

O'Brien B., Gleeson D., Mee J.F., 2005. Effect of milking frequency and feeding level on milk production, somatic cell count and reproductive performance of dairy cows over the first 20 weeks of lactation. Proc. Iri. Grassl. Agric. Res. Forum, Tullamore, Co. Offaly, 14-15 March, 31, 28.

Patton J., Kenny D.A., Mee J.F., O'Mara F.P., Wathes D.C., Cook M., Murphy J.J., 2006. Effect of milking frequency and diet on milk production, energy balance, and reproduction in dairy cows. J. Dairy Sci., 89, 1478-1487.

Pomiès D., 2002. Le robot de traite. In : Conduite à tenir : de l'animal au troupeau, du troupeau à l'animal. Journ. Nat. GTV, Tours, France, 29-31 mai, 397-400.

Pomiès D., Lefeuvre N., 2001. Impact réel d'un robot de traite sur la production laitière des vaches. Renc. Rech. Rum., 8, 263.

Pomiès D., Rémond B., 2000. Omission d'une ou deux traites par semaine sur des vaches laitières en milieu de lactation. Renc. Rech. Rum., 7, 128.

Pomiès D., Rémond B., 2002. La traite des vaches laitières une fois par jour pendant l'ensemble de la lactation : conséquences sur les performances zootechniques et la qualité du lait. Renc. Rech. Rum., 9, 195-198.

Pomiès D., Rémond B., 2007. La monotraite des vaches laitières durant trois lactations complètes successives : premiers résultats. In : $2^{\mathrm{emes}}$ Journ. Animation Scientifique du Département PHASE, 22-24 octobre, Tours, France, 200.

Pomiès D., Vimal T., Bony J., Coulon J.B., 1998. Mise en place d'un robot de traite dans une ferme expérimentale : premiers résultats obtenus à l'INRA. Renc. Rech. Rum., 5, 335-338.
Pomiès D., Martin B., Rémond B., Brunschwig G., Pradel P., Lavigne R., Hulin S., 2003. La traite une fois par jour pendant 7 semaines de vaches laitières prim'holstein et montbéliardes en milieu de lactation : performances zootechniques, qualité du lait et des fromages. Renc. Rech. Rum., 10, 81-84.

Pomiès D., Rémond B., Pradel P., 2004 Performances des vaches laitières et qualité du lait lors de la monotraite et après retour à 2 traites par jour, en fonction de la durée de cette pratique et du stade de lactation des animaux. Renc. Rech. Rum., 11, 225-228.

Pomiès D., Martin B., Chilliard Y., Pradel P., Rémond B., 2007. Once-a-day milking of Holstein and Montbéliarde cows for seven weeks in mid-lactation. Animal, 1, 1497-1505.

Portier B., Chénais F., Brocard V., 2006. Traire une fois par jour toute l'année : un revenu équivalent à certaines conditions. Cap Elevage, $6,10-13$

Rémond B., Boit M.P., 1997. Effects of milking three times in 2 days for 3 weeks in early lactation or in the declining phase on milk production in primiparous and multiparous dairy cows. Ann..Zootech., 46, 339-348.

Rémond B. Pomiès D., 2005. Once-daily milking of dairy cows: a review of recent French experiments. Anim. Res., 54, 427-442.

Rémond B., Pomiès D., 2007a. Once-daily milking of Holstein cows for one-week decreases milk yield by twenty-five percent without any carry-over effect. Liv. Sci., 110, 192-195.

Rémond B., Pomiès D., 2007b. La traite des vaches laitières une fois par jour: conséquences sur leur production, leur bien-être, leur reproduction et leur santé. Bull. GTV, 40, 83-90.

Rémond B., Coulon J.B., Nicloux M., Levieux D., 1999. Effect of temporary once-daily milking in early lactation on milk production and nutritional status of dairy cows. Ann.. Zootech., 48, 341-352.

Rémond B., Aubailly S., Chilliard Y., Dupont D., Pomiès D., Petit M., 2002. Combined effects of once-daily milking and feeding level in the first three weeks of lactation on milk production and enzyme activities, and nutritional status, in Holstein cows. Anim. Res., 51, 101-117.

Rémond B., Pomiès D., Dupont D., Chilliard Y, 2004. Once-a-day milking of multiparous Holstein cows throughout the entire lactation: milk yield and composition, and nutritional status. Anim. Res., 53, 201-212.

Rémond B., Pomiès D., Pradel P., 2005. Effet de la monotraite des vaches laitières sur leur production, selon le niveau de distribution d'aliments concentrés. Renc. Rech. Rum., 12, 229-232.

Rémond B., Pomiès D., Julien C., Pradel P., 2006. Effets de faibles écarts de temps entre les deux traites de la journée sur la quantité de lait produite et sa composition, chez la vache laitière. Renc. Rech. Rum., 13, 365-368.

\section{RICA.http://ec.europa.eu/agriculture/rica/}

Rubin B., Huchon J.C., Sabatte N. Désarménien D., Gaboriau L., Goulard L., 2006. Robot de traite, monotraite, embauche d'un salarié : impacts sur les systèmes fourragers des exploitations en Pays-de-la-Loire. Fourrages, 185, 63-72.

Salama A.A.K., Such X., Caja G., Rovai M., Casals R., Albanell E., Marin M.P., Marti A., 2003. Effects of once versus twice daily milking throughout lactation on milk yield and milk composition in dairy goats. J. Dairy Sci., 86, 16731680 .

Saumande J., 2002. Suivi de la reproduction. In : Le robot de traite : aspects techniques et économiques. Bony J., Pomiès D. (Eds), INRA Editions, Paris, France, 58-63.

Tucker C.B., Dalley D.E., Burke J.L., Clark D.A., 2007. Milking cows once daily influences behavior and udder firmness at peak and mid lactation. J. Dairy Sci., 90, 1692-1703.

Veysset P., Wallet P., Prugnard E., 2001. Le robot de traite : pour qui ? pourquoi? Caractérisation des exploitations équipées, simulations économiques et éléments de réflexion avant investissement. INRA Prod. Anim., 14, 5161.

\section{Résumé}

Depuis quelques années, les éleveurs laitiers français aspirent à une meilleure maîtrise de leur temps de travail, notamment vis-à-vis de la traite biquotidienne qui représente près de $50 \%$ de l'astreinte. C'est dans cette optique que l'INRA a évalué et/ou mis au point des outils et des conduites de traite innovants. Chez les bovins, les travaux menés sur le robot de traite ont mis en évidence une bonne adaptation des animaux à la machine et une production laitière peu modifiée, hormis une augmentation de la lipolyse et des spores butyriques du lait Mais le prix élevé du robot et son utilisation au pâturage sont un frein pour de nombreux éleveurs. A l'opposé, la traite une seule fois par jour (monotraite) est une pratique peu coûteuse, rapide à mettre en œuvre et réversible. Elle entraîne une diminution de la production laitière (de - 15 à - $46 \%$ selon l'espèce et le contexte) associée à un lait plus riche en protéines et matières grasses. L'utilisation prolongée de la monotraite pouvant entraîner une dégradation de la santé de la mamelle et de la qualité du lait, sa mise en ouvre se limite généralement à quelques semaines. La suppression de la traite du dimanche soir, la modulation des horaires de traite ou la monotraite en présence du jeune sont d'autres alternatives à la traite «classique», qui permettent de répondre en partie aux aspirations des éleveurs à une meilleure qualité de vie. Le développement de ces conduites repose en grande partie sur la possibilité de sélectionner des femelles laitières aptes à tolérer de longs intervalles entre traites. 


\begin{abstract}
Simplified milking methods in dairy herds: the end of twice-a-day milking constraints

For a few years, French dairy farmers have wanted to better manage their workload, specially regarding milking, which takes about half of their working time. To do this, INRA has tested and developed innovative milking tools and methods. In cattle, studies on robotic milking having shown that animals adapt easily to such systems, but lipolysis and butyric spore counts of milk are increased. However, the high cost of milking robots and the fact that they are difficult to use when cattle are at pasture appear as barriers for French farmers. By contrast, once-daily milking is cheap, can be implemented quickly, and is easily reversible to conventional (i.e. twice-daily) milking. Oncedaily milking results in a reduction in milk yield (from -15 to $-46 \%$ according to species and context) and an increase in protein and lipid content of milk. It shall be limited to a few weeks; otherwise, it may be detrimental to udder health and milk quality. Other alternatives to conventional milking that can improve the farmers' quality of life are the suppression of milking on Sunday evenings, changes in intervals between milkings, and once a day milking combined with calf suckling. The success of such techniques depends on the possibility to select cows that tolerate large intervals between milkings.
\end{abstract}

POMIÈS D., MARNET P.-G., COURNUT S., BARILLET F., GUINARD-FLAMENT J., RÉMOND B., 2008. Les conduites de traite simplifiées en élevage laitier : vers la levée de l'astreinte biquotidienne. INRA Prod. Anim., 21, 59-70. 